\title{
Determinación ecográfica del volumen normal de la glándula tiroides en una población pediátrica de Bogotá, Colombia
}

\author{
Marcela González ${ }^{1}$, Claudia Patricia González ${ }^{2,3}$, Álvaro Sanabria ${ }^{4}$ \\ ${ }^{1}$ Departamento de Radiología, Hospital de San José, Bogotá, D.C., Colombia. \\ 2 Departamento de Radiología, Organización Sánitas, Bogotá, D.C., Colombia. \\ ${ }^{3}$ Servicio de Radiología, Clínica Jorge Bejarano, Bogotá, D.C., Colombia. \\ ${ }^{4}$ División de Investigaciones, Fundación Universitaria de Ciencias de Salud, Bogotá, D.C., Colombia
}

Introducción. La Organización Mundial de la Salud utiliza como referencia para el tamaño normal de la glándula tiroides en niños valores obtenidos en poblaciones europeas. Sin embargo, por la variabilidad derivada de diferencias geográficas, raciales y nutricionales recomienda la obtención de valores normales para cada región.

Objetivo. Estimar el volumen normal de la glándula tiroides en niños de Bogotá, Colombia.

Materiales y métodos. Se realizó un estudio de corte transversal en niños que asistieron a la consulta de niño sano de la Clínica Jorge Bejarano de Bogotá, Colombia, entre enero y agosto de 2003. Se excluyeron los niños con antecedentes maternos o personales de enfermedad tiroidea o retardo en el desarrollo pondo-estatural. Para cada niño se registró edad, peso, talla e índice de superficie corporal. Además, se determinó el volumen de la gándula tiroides mediante ecografía, utilizando la fórmula de la elipse.

Resultados. Se incluyeron 591 niños. El promedio de los volúmenes estimados en cada grupo de edad fue de $0,63 \pm 0,2 \mathrm{~cm}^{3}$ en recién nacidos; $1,1 \pm 0,61 \mathrm{~cm}^{3}$ en lactantes; $2,2 \pm 1,3 \mathrm{~cm}^{3}$ en preescolares; $3 \pm 1,7 \mathrm{~cm}^{3}$ en escolares, y $5,7 \pm 3,15 \mathrm{~cm}^{3}$ en púberes. Se evidenció un aumento del volumen de la glándula tiroides con el incremento de la superficie corporal. Se encontró una relación lineal entre el logaritmo del volumen de la glándula tiroides y la raíz cuadrada de la superficie corporal.

Conclusión. El volumen de la glándula tiroides normal en niños de Bogotá es inferior al utilizado como referencia por la Organización Mundial de la Salud. Recomendamos utilizar los valores descritos en este estudio como parámetro local de normalidad.

Palabras clave: ultrasonografía, pediatría, glándula tiroides, Colombia, América Latina, Organización Mundial de la Salud.

Ultrasonographic estimation of the normal volume of the thyroid gland in pediatric populations.

Introduction. The World Health Organization bases its reference standards for normal thyroid volume on values derived from measurements of European children. However, WHO recommends that normal values for each region be estimated separately, given the variability as a consequence of geographic, racial, and nutritional differences.

Objective. A normal standard of thyroid volume was estimated for the pediatric population of Bogotá, Colombia.

Materials and methods. A cross-sectional study was carried-out in 591 children assessed at the Clínica Jorge Bejarano, Bogotá, Colombia, between January and August 2003. Children with maternal or personal history of thyroid disease and those with abnormal weight or height characteristics were excluded. Age, weight, and body surface index were recorded for each child.Thyroid volume was estimated by ultrasonography using the ellipse formula.

Results. The mean estimated volume for each age group was as follows: $0.6 \pm 0.2 \mathrm{~cm}^{3}$ for neonates, $1.1 \pm 0.6$ cubic centimeters for milk-fed babies, $2.2 \pm 1.3 \mathrm{cc}$ for pre-school age children, $3.0 \pm 1.7 \mathrm{cc}$ for school age children and $5.7 \pm 3.1 \mathrm{cc}$ for adolescents. Thyroid volume increases with increasing body surface. A linear relationship between the natural logarithm of the thyroid volume and the square root of the body surface was found. 
Conclusions. The normal thyroid volume for Bogotá children was smaller than the World Health Organization reference value. Values described in the current study are recommended for local use as the normal parameter.

Keywords: Ultrasonography, pediatrics, thyroid gland, Colombia, Latin America, World Health Organization.

Los rangos normales del volumen de la glándula tiroides se encuentran claramente establecidos en la población adulta. En los niños debe tenerse en cuenta que dicho volumen varía según la etapa de crecimiento y, también, como consecuencia de factores como el aporte de yodo en la dieta, entre otros. La Organización Mundial de la Salud (OMS) ha tomado como patrón de referencia del volumen de la glándula tiroides normal en niños, el establecido por estudios realizados en la población europea. Sin embargo, recomienda la realización de estudios en cada país (1), ya que el volumen de la glándula tiroides cambia de acuerdo con factores nutricionales (ingestión de yodo), geográficos (presencia de rocas sedimentarias en las vertientes de agua) y raciales locales. Esta información es de utilidad clínica y como indicador de salud pública.

Se han adelantado estudios en Estados Unidos, Brasil, Irán, Turquía, Bangladesh, Japón, Italia, Malasia, Alemania, Bélgica, Polonia y Suiza (210) que han permitido establecer los valores normales del volumen de la glándula tiroides para las respectivas poblaciones pediátricas. Hasta el momento no existían estudios realizados en la población infantil colombiana.

El presente estudio se planteó como objetivo estimar el volumen de la glándula tiroides normal de los niños de Bogotá, Colombia, estratificar dicho volumen según grupos de edad y correlacionarlo con la superficie corporal.

\section{Materiales y métodos}

Entre enero y agosto del 2003 se realizó un estudio de corte transversal para determinar el volumen

$\overline{\text { Correspondencia: }}$

Marcela González

División de Investigaciones, Fundación Universitaria de

Ciencias de la Salud, carrera 19 No. 8A - 32, Bogotá,

Colombia

Teléfono: 5998977, ext. 160; fax: 5998977, ext. 161

marceladali@yahoo.com

Recibido: 12/08/05; aceptado: 05/12/05 de la glándula tiroides por ecografía y su relación con la edad y la superficie corporal en niños colombianos sanos.

La población escogida incluyó pacientes menores de quince años que asistían a control del niño sano o que consultaron por una condición clínica aguda leve no relacionada con patología tiroidea, como infección respiratoria, enfermedad diarreica o trauma ortopédico menor en la Clínica del Niño Jorge Bejarano de Bogotá. Este es un centro pediátrico que atiende hijos de padres afiliados al sistema de seguridad social colombiano.

Se excluyeron los pacientes con antecedente de enfermedad crónica, peso o talla inferior al percentil 5 o superior al percentil 95 según las tablas de peso y talla para la edad del Instituto Colombiano de Bienestar Familiar, con antecedente de enfermedad tiroidea materna, enfermedad tiroidea, enfermedades congénitas o hallazgos ecográficos anormales diferentes del volumen durante el examen .

Las ecografías fueron practicadas por un radiólogo o un residente de radiología de último año con ecógrafo Aloka SSD 1000, utilizando un transductor de 7,5 MHz. El estudio se hizo con el paciente en decúbito supino y el cuello en extensión. Se registraron los diámetros longitudinal, transverso y antero-posterior de cada lóbulo tiroideo en milímetros. El volumen se determinó para cada lóbulo empleando la fórmula de la elipse que calcula el volumen como el producto del número pi y los diámetros longitudinal y transverso dividido por 6 (7). El volumen total se calculó sumando los valores de todos los lóbulos.

Adicionalmente se registró para cada niño la edad, el peso en kilogramos y la talla en centímetros. Utilizando los datos de peso y talla se determinó en cada caso el índice de superficie corporal (producto del peso y la talla dividido por 3.600).

Los niños se estratificaron por edad así: recién nacido (0 a 30 días), lactante ( 1 mes a 2 años), 
preescolar (3 a 6 años), escolar (7 a 10 años), púber (11 a 15 años).

Se calculó una muestra estratificada por grupo de edad de 113 pacientes para cada grupo con el programa Tamamu $\AA$, suponiendo un alfa de 0,05 y una precisión del 7\%; como medida inicial se usaron los promedios y la desviación estándar reportadas por $\mathrm{Xu}$ y colaboradores (3). Las variables categóricas se resumieron como proporciones y las variables numéricas como promedio y desviación estándar. El supuesto de normalidad de las variables se evaluó con la prueba de Shapiro-Wilks.

Finalmente, se hizo un análisis de regresión simple utilizando el volumen de la glándula tiroides y el área de superficie corporal. Dado que las dos variables no cumplían el principio de linealidad, se realizó un ajuste utilizando el logaritmo natural del volumen de la glándula tiroides y la raíz cuadrada de la superficie corporal. La formula derivada con sus intervalos de predicción del $95 \%$ se utilizó para generar los valores esperados del volumen y éstos fueron convertidos nuevamente a unidades naturales.

El estudio fue aprobado por los comités de ética de la Fundación Universitaria de Ciencias de la Salud, del Hospital de San José y de la Clínica Jorge Bejarano de Bogotá. En todos los casos se obtuvo consentimiento informado por parte de los adultos responsables de cada niño antes de su inclusión en el estudio.

\section{Resultados}

Se consideró inicialmente un total de 624 pacientes en el estudio, de los cuales se excluyeron 33 (24 por presentar peso o talla

Cuadro 1. Media y desviación estándar del peso en $\mathrm{kg}$ para cada grupo de edad.

\begin{tabular}{lccc}
\hline Grupo de edad & $\mathbf{n}$ & $\begin{array}{c}\text { Media } \\
\mathbf{( k g )}\end{array}$ & $\begin{array}{c}\text { Desviación } \\
\text { estándar }\end{array}$ \\
\hline Recién nacidos & 114 & 3,17 & 0,54 \\
Lactantes & 145 & 8,4 & 2,87 \\
Pre-escolares & 106 & 16,5 & 2,9 \\
Escolares & 114 & 27,3 & 5,8 \\
Púberes & 112 & 43,8 & 9,6 \\
\hline
\end{tabular}

inferior al percentil 5 o superior al 95 para su edad y 9 por hipotiroidismo materno). De los 591 pacientes, 303 eran hombres y 288 , mujeres (relación hombre-mujer 1,05:1); 114 estaban dentro de la categoría de recién nacidos, 145 en la de lactantes, 106 en la de preescolares, 114 en la de escolares y 112 en la de púberes.

La media del peso y la desviación estándar para cada grupo de edad se muestran en el cuadro 1. La media del volumen de la glándula tiroides y la desviación estándar para cada grupo de edad se describen en el cuadro 2. La figura 1 muestra la distribución por percentiles del volumen de la glándula tiroides según el área de superficie corporal. La figura 2 demuestra una correlación lineal entre el logaritmo del volumen de la glándula tiroides y la raíz cuadrada del área de superficie corporal, con un aumento proporcional en cada grupo y una precisión del $95 \%$. El volumen de la glándula tiroides aumentó con el incremento de la edad y del área de superficie corporal.

\section{Discusión}

Los trastornos por deficiencia de yodo se consideran de importancia en salud pública, ya que constituyen la principal causa prevenible de daño cerebral y retraso mental (11). Como consecuencia, el Fondo de las Naciones Unidas para la Infancia Unicef utiliza como marcadores de salud pública en cada país el porcentaje de hogares que consumen sal yodada y el tamaño de la glándula tiroides medido en términos de la frecuencia de bocio en niños entre 6 y 11 años de edad (12). En 1988, Unicef declaró a Colombia país libre de trastornos por deficiencia de yodo al estimar que $91 \%$ de los hogares empleaba sal correctamente yodada y que solamente $7 \%$ de

Cuadro 2. Media y desviación estándar de volumen de la glándula tiroides en $\mathrm{cm}^{3}$ para cada grupo de edad calculada con la fórmula de la elipse.

\begin{tabular}{lccc}
\hline Grupo de edad & $\mathbf{n}$ & $\begin{array}{c}\text { Media } \\
\mathbf{( c m}^{\mathbf{3}} \mathbf{)}\end{array}$ & $\begin{array}{c}\text { Desviación } \\
\text { estándar }\end{array}$ \\
\hline Recién nacidos & 114 & 0,63 & 0,2 \\
Lactantes & 145 & 1,1 & 0,61 \\
Preescolares & 106 & 2,2 & 1,3 \\
Escolares & 114 & 3,0 & 1,7 \\
Púberes & 112 & 5,7 & 3,13 \\
\hline
\end{tabular}




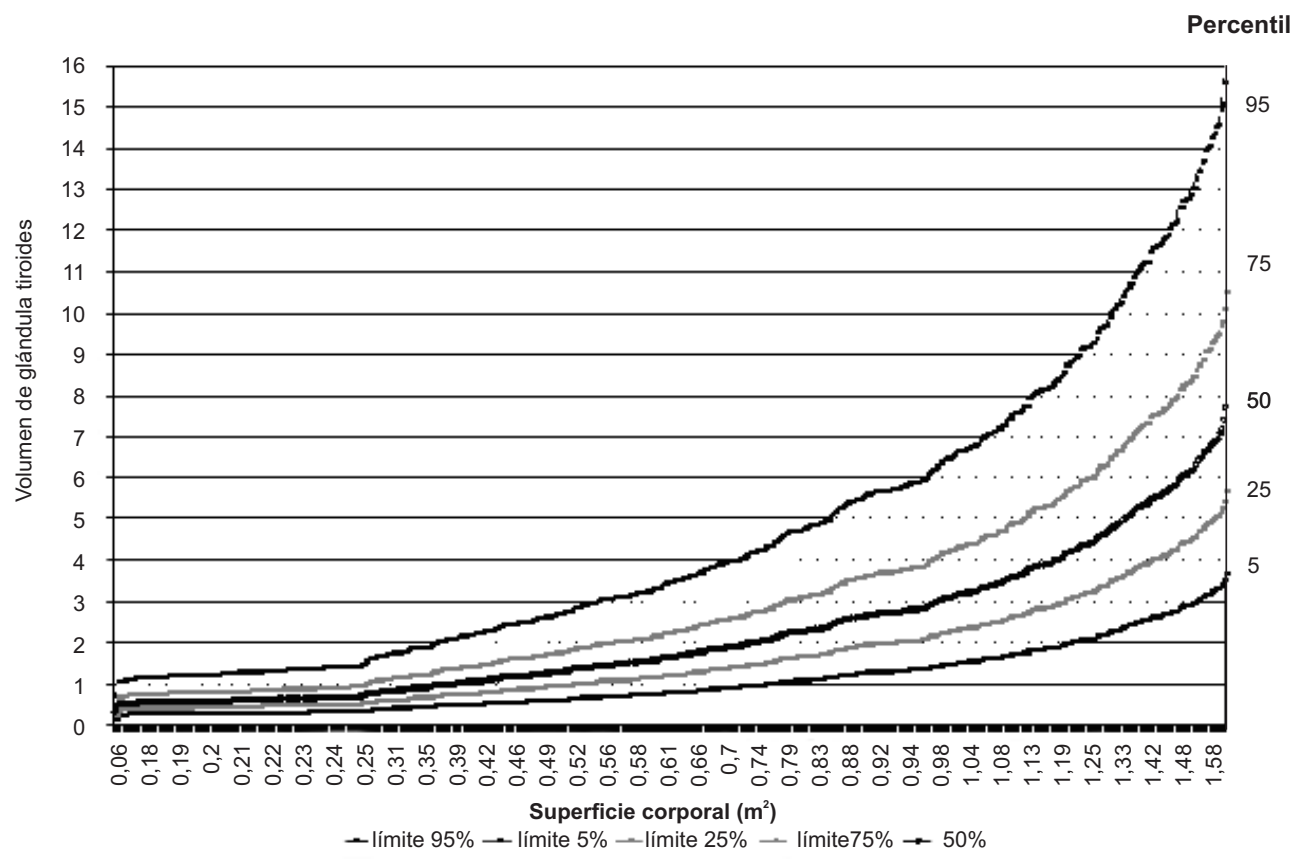

Figura 1. Relación entre el índice de superficie corporal $\left(\mathrm{m}^{2}\right)$ y el volumen de la glándula tiroides (g).

los niños entre 6 y 11 años tenía bocio (13). En 1989 , varios estudios realizados en el occidente colombiano mostraron que la ingestión de yodo era adecuada en 185 mujeres embarazadas mediante la medición de yodo urinario (14). La ingestión adecuada de yodo fue confirmada por un estudio adelantado poco después por el Instituto Nacional de Salud de Colombia en 74 municipios y 32 departamentos, que estimó una prevalencia de bocio del $7 \%$ en una muestra de 15.807 escolares y reportó una concentración de yodo en orina superior a $100 \mathrm{ug} / \mathrm{L}$ en el $92,8 \%$ de 5.971 muestras analizadas (15).

Nuestros hallazgos sugieren que el volumen de la glándula tiroides en la población colombiana evaluada es similar al descrito en la literatura para Brasil y los Estados Unidos (2,3). En contraste, el volumen de la glándula tiroides en niños bogotanos sanos resultó inferior al estándar de referencia europeo y al reportado para otros países del mundo $(1,4-6)$. Estos resultados son explicables en parte por las diferencias en el aporte nutricional de yodo para cada región geográfica. Según la Unicef, la proporción de familias que

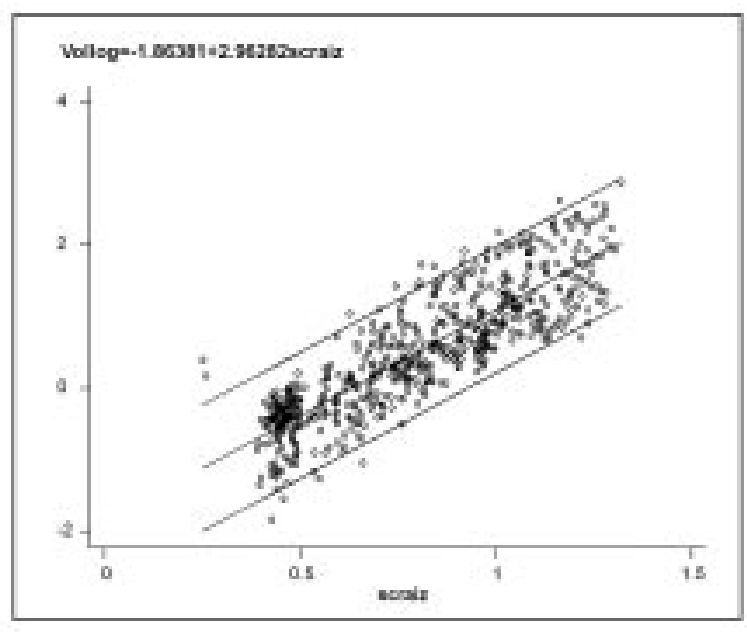

Figura 2. Correlación entre el volumen tiroideo (logaritmo) y el área de superficie corporal (raíz cuadrada).

consume sal yodada en los países de Europa central y del este, Turquía, Irán y Brasil es del $25 \%, 18 \%, 82 \%$ y $95 \%$, respectivamente (12). De manera predecible, un estudio realizado en Turquía mostró que el 34\% de la población presentaba volúmenes de la glándula tiroides en 
niños por encima de los límites superiores de referencia europeos (5). En cambio, estudios realizados en Brasil mostraron un volumen de la glándula tiroides para niños sanos expuestos a una dieta rica en yodo relativamente inferior al descrito por estudios europeos e iraníes $(1,2,6)$.

Además de su importancia como indicador de salud pública, el tamaño de la glándula tiroides tiene importancia clínica. Estudios realizados en el occidente colombiano indican que el tamaño de la glándula tiroides puede aumentar como consecuencia de factores independientes de la ingestión de yodo $(14,16,17)$. Entre los posibles agentes bociógenos se reconocen la composición geológica y la contaminación bacteriana de las vertientes de agua $(16,17)$. Hasta el $80 \%$ de la variación geográfica en la prevalencia de bocio de una región a otra de Colombia se puede explicar por estos factores (16). Las enfermedades tiroideas de distinto tipo también pueden generar cambios en el tamaño de la glándula tiroides (18). Resulta, entonces, relevante para el clínico conocer los parámetros de normalidad de la glándula tiroides para la población pediátrica correspondiente a su región.

Presentamos el primer estudio que estima el volumen de la glándula tiroides normal de la población pediátrica bogotana. Se presentan los valores según el grupo de edad y los percentiles según el área de superficie corporal. Nuestros resultados son útiles desde el punto de vista clínico y como indicadores indirectos de salud pública. Consideramos preferible el empleo de estos valores como referencia local que el de valores definidos en otras partes del mundo.

\section{Agradecimiento}

A Carlos Díaz Granados de la División de Investigaciones de la Fundación Universitaria de Ciencias de la Salud, Bogotá, Colombia, por su colaboración en la presentación de resultados y la redacción del manuscrito.

\section{Financiación}

Este trabajo fue autofinanciado.

\section{Conflicto de Intereses}

Los autores no tienen conflictos de intereses.

\section{Referencias}

1. ThyroMobil Study Group World Health Organization, International Council for Control of lodine Deficiency Disorders. Recommended normative values for thyroid in children aged -15 years. Bull World Health Organ 1997;75:95-7.

2. Rossi A, Tomimori E, Camargo R, Medeiros-Neto G. Determination of thyroid volume by sonography in healthy Brazilian schoolchildren. J Clin Ultrasound 2002;30:226-31.

3. Xu F, Sullivan K, Houston R, Zhao J, May W, Maberly G. Thyroid volume in US and Bangladeshi schoolchildren: comparison with European schoolchildren. Eur J Endocrinol 1999;140:498-504.

4. Semiz S, Senol U, Bircan O, Gumuslu S, Bilmen S, Bircan I. Correlation between age, body size and thyroid volume in an endemic area. J Endocrinol Invest $2001 ; 24: 559-63$.

5. Semiz S, Senol U, Bircan O, Gumuslu S, Akcurin S, Bircan I. Thyroid gland volume and urinary iodine excretion in children 11 years old in an endemic area. $\mathrm{J}$ Pediatr Endocrinol Metab 2000;13:245-51.

6. Azizi F, Delshald H, Mehrabi Y. Thyroid volumes in schoolchildren of Tehran: Comparison with European schoolchildren. J Endocrinol Invest 2001;24:756-62.

7. Ueda D. Sonographic measurement the volume of thyroid gland in healthy children. Acta Paediatr Jpn 1989;31:352-4.

8. Muller-Leisse C, Troger J, Khabirpour F, Pockler C. Normal values of thyroid gland volume. Ultrasound measurements in schoolchildren 7 to 20 years of age. Dtsch Med Wochenschr 1988;113:1872-5.

9. Mahmud N. Measurement of thyroid volume in children using a portable ultrasound machine. Asia Pac J Public Health 2001;13:36-9.

10. Lupoli G, Russo D, Fittipaldi MR, Vitale G, Napodano A, Pagliuca A et al. Evaluation of goiter endemia by ultrasound in schoolchildren in Val Sarmento (Italy). J Endocrinol Invest 1999;22:503-7.

11. Pretell EA, Aguirre A, Guell R, Canelos P, Higa AM, Cevallos JL et al. Consenso sobre los desórdenes por deficiencia de yodo en Latinoamérica. Criterios de evaluación y monitoreo para su erradicación sostenida. Rev Cubana Endocrinol 1999;10:146-56.

12. UNICEF. Tablas estadísticas del estado mundial de la infancia, 1998. [Consultado 2005, octubre 3]. Disponible en: http://www.unicef.org/sowc98/tab2.htm.

13. UNICEF/Colombia. Situación de la infancia. Nutrición infantil y micronutrientes. [Consultado 2005, octubre 3]. Disponible en: http://www.unicef.org.co/05-nut.htm.

14. Gaitan E, Cooksey RC, Meydrech EF, Legan J, Gaitan GS, Astudillo $\mathbf{J}$ et al. Thyroid function in 
neonates from goitrous and nongoitrous iodine-sufficient areas. J Clin Endocrinol Metab 1989;69:359-63.

15. Ministerio de Salud, Instituto Nacional de Salud, Instituto Colombiano de Bienestar Familiar, UNICEF, Asociación Colombiana de Endocrinología. Prevalencia de los desórdenes por deficiencia de yodo, Colombia, 1994-1996. Bogotá: Instituto Nacional de Salud; 2001.

16. Gaitan E. Endemic goiter in Western Colombia. Ecol Dis 1983;2:295-308.
17. Meyer JD, Gaitan E, Merino H, DeRouen T. Geologic implications in the distribution of endemic goiter in Colombia, South America. Int J Epidemiol 1978;7:2530.

18. Bettendorf $\mathbf{M}$. Thyroid disorders in children from birth to adolescence. Eur J Nucl Med Mol Imaging 2002;29 (Suppl 2):439-46.
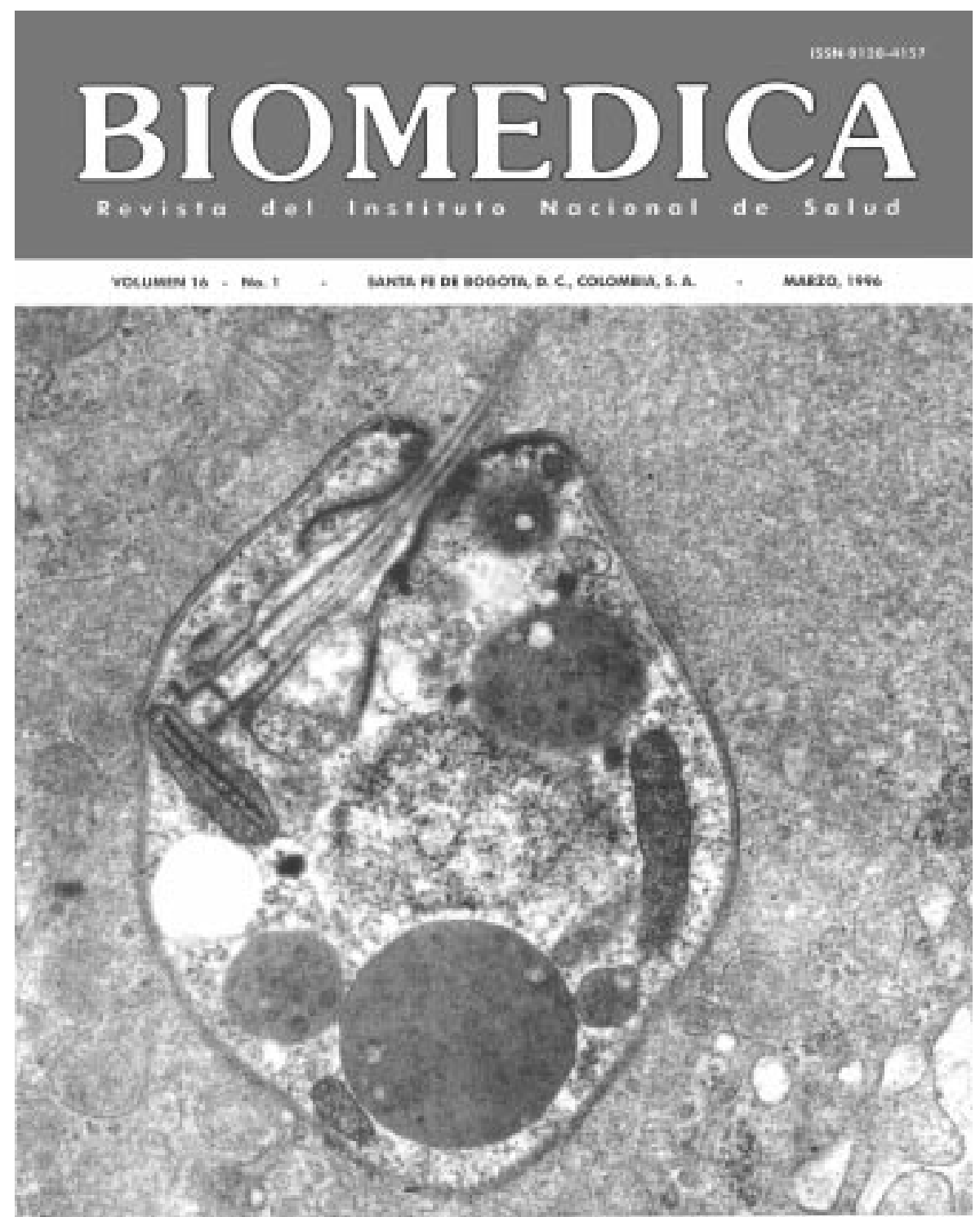